\title{
Accurate Entry Point for Tibial Nailing with SIGN Nail in Asians: A Cadaveric Study
}

Tanawat Vaseenon ${ }^{1}$, Sirichai Luevitoonvechkij ${ }^{1}$, Wittaya Akkaraatimart ${ }^{2}$ and Anupong Laohapoonrungsee ${ }^{1}$

${ }^{1}$ Department of Orthopaedics, Faculty of Medicine, Chiang Mai University, Chiang Mai, Thailand

${ }^{2}$ Orthopaedics, Photaram Hospital, Rachaburi, Thailand

\begin{abstract}
Background: Tibial nailing is a standard treatment of tibial fracture. Placing the nail in the wrong position will result in poor fracture alignment and potentially damage to cortical bone. But the exactly entry point of this technique in Thai people has never been studied. In Chiang Mai University hospital, a tibial SIGN nail is commonly used in
\end{abstract} tibial shaft fracture.

Objective: To identify the accurate entry point for tibial nailing with tibial SIGN nail, defined as the point which will provide adequate fracture alignment.

Design: Cadaveric study

Methods: Twelve cadavers with attached knee joints underwent tibial nailing with tibial SIGN nails. After placement of the nail, the specimens underwent osteotomies at the level of 10 centimeters distal to the articular surface. Multiple entry points were tested to determine fracture alignment. Medial-lateral and anterior-posterior displacements from plain radiography were recorded for these various points of entry.

Results: In coronal plane, the entry point at the sixty percent from medial edge of tibial plateau was identified as minimizing the medial-lateral displacement of the tibial shaft following fracture. In sagittal plane, the entry point of 20 $\mathrm{mm}$ posterior to the tibial tubercle resulted in the least anterior-posterior displacement.

Conclusion: When tibial nailing with tibial SIGN nail was used, the entry point of $20 \mathrm{~mm}$ posterior to the tibial tubercle and sixty percent of the total distance from medial tibial plateau provided the accurate balance of fracture reduction.

Keywords: Entry point; Tibial nailing; SIGN nail; Tibia fracture

\section{Introduction}

Tibial shaft fracture is a common orthopaedic and trauma condition. Incidence of tibial fracture is average 26 in 100,000 per year at the age of 15 to 19 years. It is more common in US that is 109 in 100,000 per year [1]. Operative treatment options are external fixation, open reduction and internal fixation with plate and screws or intramedullary nailing. Intramedullary nailing is widely accepted as a standard treatment for long bone fracture including tibial shaft fracture with benefits of less soft tissue injury, biologic preservation and advantage of biomechanics.

The crucial part for IM nailing is the entry point. It should be in the midpoint of the tibial shaft both in anteroposterior and mediolateral plane. An inappropriate entry point can cause malalignment of fracture reduction, difficulty in nail insertion or iatrogenic fracture. The optimal entry point should be in the line of the tibial medullary canal and should not damage the articular cartilage of the tibial joint and patellar ligament.

The entry point has been reported to be at the medial aspect of the tibial tubercle in coronal plane [2]. O Lembcke et al. revealed that too lateral entry point caused a varus deformity and too medial entry point created a valgus deformity [3]. Hernigou P, Cohen D [4] revealed that $2.5 \mathrm{~mm}(+/-1.8 \mathrm{~mm})$ lateral to the centre of the tibial tubercle is an ideal entry point. Too distal entry point leads to proximal posterior cortex breakage.

Timothy et al. [5] reported the anatomical safe zone for superolateral tibial portal placement was found to average only 22.9 millimeters wide and as narrow as 12.6 millimeters and the important point is prevention of damages to the intraarticular structures during portal placement and nail insertion.

The medialized nail entry point caused posteriorly and laterally directed nail insertion angle potentially leading to malalignment. The radiographic study of Freedman and Johnson reported that malalignment was commonly found in proximal third fracture of the tibial shaft (58\%), following by distal third (8\%) and middle third fracture (7\%) [6].

However, there is no study about the entry point for tibial nailing using SIGN nail in Asian population. In Chiang Mai University hospital, the tibial SIGN nail is commonly used as non-locking and interlocking intramedullary nail due to appropriate quality and cost that has been reported in the previous literature [7]. Since the tibial SIGN nail design is different from the other tibial nail models. Our study aims to identify an optimal entry point for SIGN nail in Thai patients.

\section{Methods}

Twelve cadaveric legs from seven male and five female with an

*Corresponding author: Tanawat Vaseenon, Department of Orthopaedics, Faculty of Medicine, Chiang Mai University, Chiang Mai, Thailand, Tel. 6653945544; Fax: 6653946442; E-mail: tvaseenon@yahoo.com

Received April 19, 2012; Accepted May 10, 2012; Published May 13, 2012

Citation: Vaseenon T, Luevitoonvechkij S, Akkaraatimart W, Laohapoonrungsee A (2012) Accurate Entry Point for Tibial Nailing with SIGN Nail in Asians: A Cadaveric Study. J Trauma Treat 1:135. doi:10.4172/2167-1222.1000135

Copyright: (c) 2012 Vaseenon T, et al. This is an open-access article distributed under the terms of the Creative Commons Attribution License, which permits unrestricted use, distribution, and reproduction in any medium, provided the original author and source are credited. 
average age of 43.8 years (range 28-57 years) at the time of death were used in this study. All specimens had no obvious injury or underwent an operation around the knee area. Closed antegrade tibial nailing was performed by one of the authors (T.V.) and the procedure was as follows.

\section{Nail selection}

The specimens were taken for radiography along with three sizes of nail ranging from $9-11 \mathrm{~mm}$ in diameter. The medullary canal and the diameter of nail were compared and the appropriate nail diameter was chosen. Measuring the distance from joint line of the knee to the medial malleolus chose the length of the nail.

\section{Tibial nailing}

The cadaver was set in supine position with 90 degree knee flexion. The chosen nail was inserted into the tibial bone by transpatellar tendon approach. The nail was impacted $5-10 \mathrm{~mm}$. below the joint surface and two proximal locking screws at the proximal tibia were applied through the aiming guide. The distal part of the nail was fitted into the medullary canal of the distal shaft of the tibia.

\section{Tibial osteotomy}

The shaft of the tibia was osteotomized at $10 \mathrm{~cm}$ below the articular surface by gigli saw (Figure 1).

\section{Measurement of the fracture displacement}

After osteotomy, the specimens were taken for sagittal and coronal plane radiography. The fracture steps off in both planes were measured.

\section{Arthrotomy}

Arthrotomy of the knee joint was done to evaluate the distance from the center of the nail to the tibial tubercle and the center of the tibial plateau (Figure 2).

For each radiographic and anatomical measurement, the sagittal and coronal step offs were calculated by using the regression analysis. They were considered to represent the relative contribution of the medial-lateral and anterior-posterior displacement to the optimal

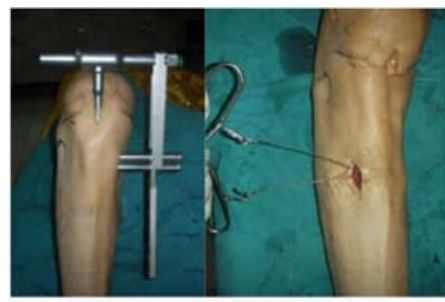

Figure 1: Tibial SIGN nail insertion and osteotomy point of the tibia.

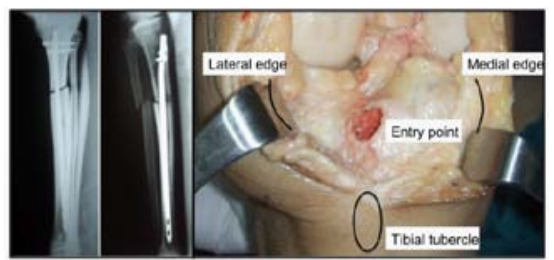

Figure 2: The anteroposterior and lateral radiographs of the specimen after osteotomy and the proximal tibial surface with the entry point of the tibial SIGN nail.

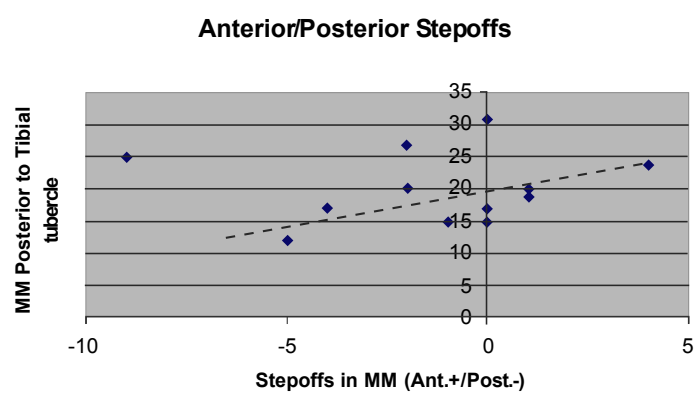

Figure 3: The relation of the entry point of the nail and anterior-posterior step offs; the optimal entry point was at $20 \mathrm{~mm}$ posterior to the tibial tubercle.

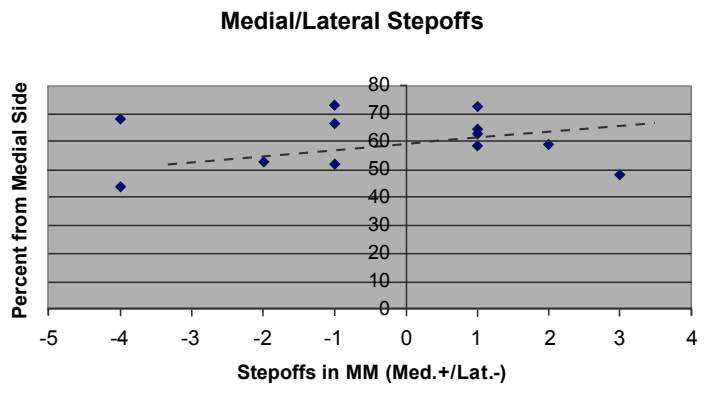

Figure 4: The relation of the entry point of the nail and the medial-lateral step offs; the optimal entry point is around $60 \%$ from the medial edge of the tibial articular surface.

entry point of the tibial nail. This analysis was applied individually to each specimen. The statistical analysis was performed with STATA software (version 10.0, Statacorp LP, college station, Tx, USA)

\section{Results}

From all specimens, Table 1, entry points were no damages to the surrounding joint surface. In sagittal plane, the entry point was at 12-27 $\mathrm{mm}$ posterior to the tip of the tibial tubercle and produced $0-9$ $\mathrm{mm}$ step offs. Each of the AP step offs and their respective positions in millimeters posterior to the tip of the tibial tubercle were plotted and analyzed by linear regression analysis. The entry site at $20 \mathrm{~mm}$ posterior to the tibial tubercle showed least step off of the fracture (Figure 3).

In the coronal plane, the distance was measured from medial and lateral edge of the articular cartilage to the center of the nail in millimeters. These values were converted into a percentage from the medial edge of articular cartilage, ranging from $43.7-72.8 \%$ of the distance between the medial and lateral articular edge. The tibial displacement ranged from $4 \mathrm{~mm}$ medially to $3 \mathrm{~mm}$ laterally. The mediolateral step offs and the corresponding distance percentages were plotted and analyzed by linear regression analysis. The statistical analysis revealed the least displacement of the osteotomy site at the point of 60 percent from the medial edge of tibial articular surface (Figure 4).

\section{Discussion}

Varieties of implants are available for fracture stabilization. Either plate or intramedullary nail is accepted in tibial shaft fixation. The biomechanical properties and the closed technique of nail insertion allow the nail to be more effective than the plate. Most of the nailing complications are avoidable by selecting a proper site of insertion [8] 
Citation: Vaseenon T, Luevitoonvechkij S, Akkaraatimart W, Laohapoonrungsee A (2012) Accurate Entry Point for Tibial Nailing with SIGN Nail in Asians: A Cadaveric Study. J Trauma Treat 1:135. doi:10.4172/2167-1222.1000135

Page 3 of 3

\begin{tabular}{|c|c|c|c|c|c|c|c|c|c|c|c|c|}
\hline & Trial 1 & Trial 2 & Trial 3 & Trial 4 & Trial 5 & Trial 6 & Trial 7 & Trial 8 & Trial 9 & Trial 10 & Trial 11 & Trial 12 \\
\hline Tip of the tibial tubercle $(\mathrm{mm})$ & 20 & 17 & 25 & 20 & 15 & 19 & 17 & 27 & 24 & 31 & 12 & 15 \\
\hline Medial tibial plateau (mm) & 50 & 35 & 51 & 40 & 38 & 38 & 51 & 40 & 50 & 45 & 32 & 37 \\
\hline Lateral tibial plateau (mm) & 35 & 45 & 19 & 37 & 40 & 34 & 28 & 28 & 35 & 27 & 15 & 14 \\
\hline $\begin{array}{l}\text { Step off anterior/posterior } \\
(\mathrm{mm})\end{array}$ & $2 \mathrm{~mm}(\mathrm{P})$ & $4 \mathrm{~mm}(\mathrm{P})$ & $9 \mathrm{~mm}(\mathrm{P})$ & $1 \mathrm{~mm}(\mathrm{~A})$ & $0 \mathrm{~mm}$ & $1 \mathrm{~mm}(\mathrm{~A})$ & $0 \mathrm{~mm}$ & $2 \mathrm{~mm}(\mathrm{P})$ & $4 \mathrm{~mm}(\mathrm{~A})$ & $0 \mathrm{~mm}$ & $5 \mathrm{~mm}(\mathrm{P})$ & $1 \mathrm{~mm}(\mathrm{P})$ \\
\hline Step off medial/lateral (mm) & $1 \mathrm{~mm}(\mathrm{M})$ & $4 \mathrm{~mm}(\mathrm{~L})$ & $1 \mathrm{~mm}(\mathrm{~L})$ & $1 \mathrm{~mm}(\mathrm{~L})$ & $3 \mathrm{~mm}(\mathrm{M})$ & $2 \mathrm{~mm}(\mathrm{~L})$ & $1 \mathrm{~mm}(\mathrm{M})$ & $2 \mathrm{~mm}(\mathrm{M})$ & $1 \mathrm{~mm}(\mathrm{~L})$ & $1 \mathrm{~mm}(\mathrm{M})$ & $4 \mathrm{~mm}(\mathrm{~L})$ & $1 \mathrm{~mm}(\mathrm{M})$ \\
\hline$\%$ from medial side & 58.8 & 43.7 & 72.8 & 51.9 & 48.7 & 52.8 & 64.5 & 58.8 & 58.8 & 62.5 & 68 & 72.5 \\
\hline Tibia (left or right) & right & left & right & left & right & left & right & left & right & left & right & left \\
\hline
\end{tabular}

Table 1: Data regarding entry point and step offs in the anterior-posterior and medial-lateral directions of 12 cadavers.

The bony anatomy of Asians differs from that of western populations in both size and morphology such as the coronal plane of proximal tibia is more varus and the angle between the anterior shaft of the tibia and the slope of the tibial plateau in the sagittal plane is higher in Asians $[9,10]$. These affect the accuracy of the entry point of tibial SIGN nail.

The ideal entry point for tibial nailing had been reported by several authors [2-6]. Samuelson MA et al. [2] reported to be at the medial aspect of the tibial tubercle in coronal plane. In the study of Hernigou $\mathrm{P}$ and Cohen D [4] they revealed that $2.5 \mathrm{~mm}(+/-1.8 \mathrm{~mm})$ lateral to the centre of the tibial tubercle is an ideal entry point. Kuntscher and Gross-Kempf nails was used in this study. In our study, the entry point of the tibial SIGN nail was slightly different from their recommendation that was at the sixty percent from medial side of the tibial articular in the coronal plane and $20 \mathrm{~mm}$ posterior to the tip of the tibial tubercle in the sagittal plane. Our result correlated with the previous studies that reported the coronal bowing of the tibia in Asians population is higher than that of western populations, so the suitable entry point of the tibia is more laterally to avoid angular deformity in coronal plane [11]. Moreover, our result showed the entry point was $20 \mathrm{~mm}$ posterior to the tip of the tibial tubercle in sagittal plane that is different from other nails [4]. This might be the angle of the tibial SIGN nail is high, and then placing of the entry point was slightly closer to the tip of the tibial tubercle.

Principle of nail placement is insertion the nail in the medullary canal without malalignment. Too distal entry point must be avoid as reported by Hernigou P and Cohen D [4] that may lead to proximal posterior cortex breakage during operation. In addition, too lateral entry point can cause varus malalignment and too medial entry point leads to valgus malalignment as revealed by $\mathrm{O}$ Lembcke et al. [3]. In our study, we found that too posterior and too anterior entry point lead to anterior and posterior displacement, respectively. We also found that too lateral entry point caused medial displacement, leading to varus deformity and too medial entry point caused lateral displacement, leading to valgus deformity.

From the statistical analysis, no relationship between the AP step offs and the distance of the entry point to the tibial tubercle was demonstrated by a regression coefficient of $R R=0.127, p=0.813$. It might be a cause of the Herzog angle of the tibial nail that provides more stability to the fixation construct. In addition, the mediolateral step offs and the distance from the medial articular cartilage showed weak relationship with a regression coefficient of $R R=0.306, p=0.824$. The usage of human specimens in the experiment may decrease the coefficient. Difference in bone side, size and width of the medullary canal including small numbers of the specimens were possible factors affecting the results that were our limitation of the study.

Although there was no strong relationship between steps off and entry point of the tibial SIGN nail but there was a trend that when tibial nailing with tibial SIGN nail was used, the accurate entry point of 20 $\mathrm{mm}$ posterior to the tibial tubercle and sixty percent of percent from medial side provides the accurate balance of fracture reduction.

\section{References}

1. Court-Brown CM, McBirnie J (1995) The epidemiology of tibial fractures. J Bone Joint Surg Br 77: 417-421.

2. Samuelson MA, McPherson EJ, Norris L (2002) Anatomic assessment of the proper insertion site for a tibial intramedullary nail. J Orthop Trauma 16: 23-25

3. Lembcke O, Ruter A, Beck A (2001) The nail-insertion point in unreamed tibia nailing and its influence on the axial malalignment in proximal tibial fractures. Arch Orthop Trauma Surg 121: 197-200.

4. Hernigou $P$, Cohen D (2000) Proximal entry for intramedullary nailing of the tibia. The risk of unrecognised articular damage. J Bone Joint Surg $\mathrm{Br}$ 82: 3341.

5. McConnell T, Tornetta P 3rd, Tilzey J, Casey D (2001) Tibial portal placement: the radiographic correlate of the anatomic safe zone. J Orthop Trauma 15: 207-209.

6. Freedman EL, Johnson EE (1995) Radiographic analysis of tibial fracture malalignment following intramedullary nailing. Clin Orthop Relat Res: 25-33.

7. Vaseenon T, Luevitoonvechkij S, Phongdara K, Laohapoonrungsee A (2010) Optimal Entry Point for Retrograde Femoral Nailing with Tibial SIGN Nail in Asians: a Cadaveric Study. The Thai Journal of Orthopaedic Surgery 34: 1-5.

8. Morgan E, Ostrum RF, DiCicco J, McElroy J, Poka A (1999) Effects of retrograde femoral intramedullary nailing on the patellofemoral articulation. $J$ Orthop Trauma 13: 13-16.

9. Chiu KY, Zhang SD, Zhang GH (2000) Posterior slope of tibial plateau in Chinese. J Arthroplasty 15: 224-227.

10. Tang WM, Zhu YH, Chiu KY (2000) Axial alignment of the lower extremity in Chinese adults. J Bone Joint Surg Am 82-A: 1603-1608.

11. Yau WP, Chiu KY, Tang WM, Ng TP (2007) Coronal bowing of the femur and tibia in Chinese: its incidence and effects on total knee arthroplasty planning. $J$ Orthop Surg (Hong Kong) 15: 32-36. 